\title{
CARACTERÍSTICAS HIDROGRÁFICAS DEL SISTEMA LAGUNAR LOS MICOS-QUEMADA EN EL CARIBE HONDUREÑO
}

\author{
Carlos L. Brenes Rodríguez ${ }^{1 *}$, Rosario Benavides Morera ${ }^{1}$ y Daniel Ballestero ${ }^{l}$
}

\begin{abstract}
RESUMEN
Auspiciado por el Proyecto PREPAC (Plan Regional de Pesca y Acuicultura Continental) se realizó un levantamiento hidrográfico en 27 estaciones en el sistema lagunar Los Micos-Quemada en el litoral Caribe de Honduras. Los muestreos mensuales se extendieron de octubre del 2005 a mayo del 2006 y se determinaron la temperatura, el pH, el oxígeno disuelto, la salinidad y la turbidez. La temperatura superficial osciló entre los 24.2 y $31.8^{\circ} \mathrm{C}$, con variaciones temporales extremas del orden de los $7^{\circ} \mathrm{C}$. Los valores más bajos se ubican al final y principio de año (octubre-febrero). La salinidad superficial presenta un marcado ciclo estacional, con valores superiores a 12 durante los meses secos en prácticamente todo el cuerpo de agua, mientras que en la época lluviosa (octubre a febrero) están alrededor de 6. Los valores de $\mathrm{pH}$ encontrados para el período de estudio se ubicaron entre los 7.8 y 8.7. Los valores más altos se localizan en la zona central del sistema lagunar y los más bajos en las zonas cercanas a las orillas. Los máximos valores de turbidez se observan en febrero y diciembre. La zona con aguas más claras se localiza en la región cercana a la Barra de Miami en el lado noroeste de la laguna. El oxígeno superficial varió entre $5.0 \mathrm{mg} / \mathrm{L}$ y $7.6 \mathrm{mg} / \mathrm{L}$ durante los meses de octubre a febrero. La variación temporal del oxígeno disuelto muestra que los máximos y mínimos para todo el período de estudio se localizaron en octubre y mayo, respectivamente.
\end{abstract}

Palabras claves: Laguna costera, hidrografía, Honduras.

\begin{abstract}
A hydrographic survey funded by PREPAC (Plan Regional de Pesca y Acuicultura Continental) was carried out in the Micos-Quemada lagoon system in the Caribbean coast of Honduras. From October 2005 to May 200627 sampling stations were occupied to measure $\mathrm{pH}$, dissolved oxygen, salinity, and turbidity. Surface temperatures were between 24.2 and $31.8^{\circ} \mathrm{C}$ during the sampling period, with extreme temporal variations close to $7^{\circ} \mathrm{C}$. Minimum temperatures were observed between October and February. Surface salinity showed a clear seasonal cycle, with values greater than 12 during the dry months virtually over the entire body of water, while during the rainy season (October to February) salinity values were around 6. Values for $\mathrm{pH}$ were between 7.8 and 8.7 during the study. The highest $\mathrm{pH}$ values were found in the central area of the lagoon system and the lowest in the areas close to the banks, likely due to the degradation of organic matter and to the contribution from the tributary. Maximum turbidity was observed in February and December, while the area with clearest water was located near the bar. Surface oxygen concentration varied between 5.0 and $7.6 \mathrm{mg} / \mathrm{L}$ from October to February. Maximum and minimum values of dissolved oxygen were observed in October and May, respectively.
\end{abstract}

Keywords: Coastal lagoon, hydrography, Honduras.

1 Laboratorio de Oceanografía y Manejo Costero, Universidad Nacional, Heredia, Costa Rica. Apdo 86-3000. E-mails: cbrenes@una.ac.cr*,rbmorera@gmail.com,dab2@una.ac.cr.

Recibido 03-VI-2009

Aceptado 06-X-2009

DOI: http://dx.doi.org/10.15359/revmar.1.12

Rev. Mar. y Cost. ISSN 1659-455X. Vol. 1. 215-229, Diciembre 2009. 


\section{INTRODUCCIÓN}

Como en otros lugares también en América Central, las lagunas costeras han jugado un papel importante en el origen y la evolución de actividades como la pesca y la acuicultura. La importancia de las lagunas costeras y estuarios para la ecología y las actividades humanas depende en gran medida de la razón de intercambio entre estos cuerpos costeros y el océano adyacente. Este intercambio determina su habilidad para sustentar la vida marina y su capacidad para soportar las actividades inherentes al desarrollo urbano de sus litorales sin degradarse. El intercambio es forzado principalmente por procesos físicos en el interior de la cuenca: viento, mareas y densidad. El comportamiento de muchas variables en su interior es además modulado por las condiciones dinámicas y las características propias de la región costera inmediata.

Las lagunas costeras constituyen un conjunto de ambientes naturales que se desarrollan en la interfase continente-océano. Estos cuerpos de agua corresponden a sectores de tránsito y de mezcla de energía, agua y sedimento procedentes tanto del continente como del mar. Ubicados en sectores con fuerte dinámica marina, son ambientes sujetos a cambios rápidos en sus características tanto morfológicas como ambientales. Son ecosistemas de gran biodiversidad, pero a su vez son extremadamente frágiles, por lo cual el asentamiento del hombre en estos sitios es siempre delicado.
El sistema lagunar costero integrado por las lagunas Los Micos (lagunu babunu en lengua garífuna o laguna de los monos) y Quemada (lagunu gudal) se ubica en el Municipio de Tela, Departamento de Atlántida, en el litoral Caribe de Honduras (Figura 1). El rango promedio de mareas en la zona es del orden de los $25 \mathrm{~cm}$, la primera de ellas posee un espejo de agua de $44.77 \mathrm{~km}^{2}$, con una profundidad promedio de $2.1 \mathrm{~m}$, mientras que la Laguna Quemada se extiende en forma paralela a la Bahía de Tela en un área aproximada de $3.03 \mathrm{~km}^{2}$, con una profundidad promedio de $1.2 \mathrm{~m}$ (PREPAC, 2006).

La pesca artesanal es considerada una actividad de importancia significativa para las comunidades en las riberas de este cuerpo de agua. Se estima que un total de 370 pescadores, permanentes y temporales, faenan en sus aguas utilizando diferentes métodos y artes de pesca (PREPAC, 2006). Por otra parte, el turismo constituye una importante fuente de ingresos para la zona, cuyo desarrollo se ha incrementado de manera significativa en los últimos años. Este sistema lagunar forma parte de una de las áreas protegidas más importantes del país por su diversidad biológica, belleza escénica y riqueza cultural, como es el Parque Nacional Jeannette Kawas (PROLANSATE, 2004).

Desde el punto de vista ambiental, la unidad hidrológica de la Laguna Los Micos ha venido sufriendo una 
Figura 1

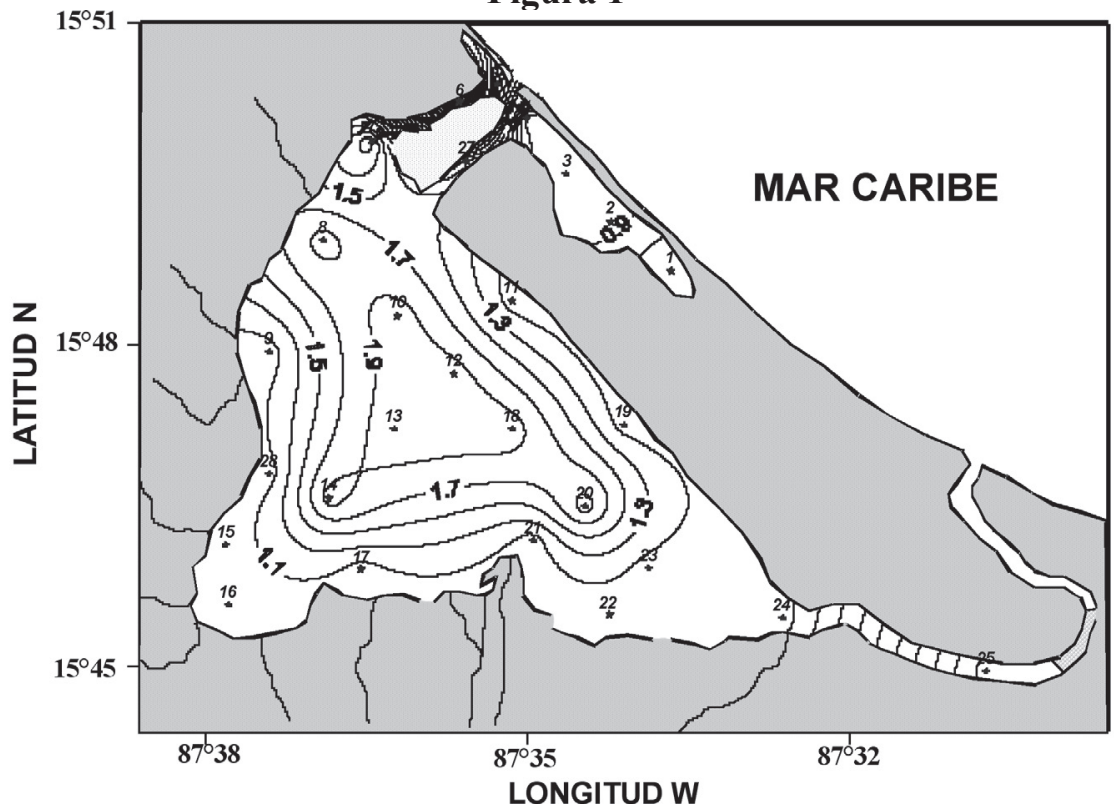

Posición de las estaciones hidrográficas y batimetría (m).

Location of hydrographic stations and bathymetry $(\mathrm{m})$.

acelerada y visible degradación que ha afectado los procesos naturales de este cuerpo de agua. Estos procesos se relacionan con la deforestación de las cuencas, el aumento en el azolvamiento de los cuerpos de agua superficiales y la extracción de recursos minerales en el lecho de los ríos, entre otros. El excesivo aporte de sedimentos llevados por el río San Alejo a la laguna ha provocado que en su desembocadura se forme una península (El Marión) de aproximadamente 200 hectáreas cubiertas por vegetación típica de otros ecosistemas (sauce, camalote) diferentes al lagunar. La sedimentación ha disminuido la profundidad y el espejo de agua de la lagu- na por el deterioro en la parte alta de la cuenca (PREPAC, 2006).

Hasta los años ochenta, el sistema lagunar Los Micos-Quemada estaba conectado al mar por dos bocas o barras temporales, la Barra de Tornabé

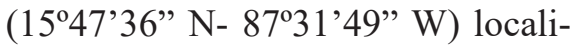
zada en la parte noreste de la laguna y la Barra de Miami en la parte noroeste $\left(15^{\circ} 50^{\prime} 33^{\prime \prime} \mathrm{N}-8^{\circ} 35^{\prime} 48^{\prime \prime}\right.$ W), produciendo un flujo de entrada y salida de agua marina con patrones de circulación propios de este tipo de ecosistemas lagunares costeros (PREPAC, 2006).

En la década de los noventa, el canal de Tornabé estuvo obstruido temporalmente, debido a procesos de 
azolvamiento provocados por los ríos La Esperanza y San Alejo (Figura 1), $\mathrm{y}$ al crecimiento de plantas acuáticas. Esta situación fue aprovechada en 1993 para efectuar el relleno total y la construcción de la carretera de acceso al proyecto de Bahía de Tela y los caseríos aledaños, al cerrarse una de las conexiones directas que la laguna tenía al mar se modificaron las características hidrodinámicas dentro de la Laguna Los Micos (Instituto Hondureño de Turismo, 1993).

La zona noroccidental del Caribe presenta un clima de carácter tropical (Gray, 1993), en la cual los altos valores de radiación total incidente y las altas temperaturas de sus aguas superficiales $\left(>27^{\circ} \mathrm{C}\right)$ prevalecen durante la mayor parte del año.

Los vientos dominantes en esta región son los alisios, casi persistentes durante todo el año, con velocidades promedio de 3-8 m/s (Gallegos, 1996). El régimen climático de la parte septentrional del Caribe está bajo la influencia de los desplazamientos norte-sur de la Zona de Convergencia Intertropical (ZCIT). Cuando esta zona se desplaza hacia su posición más al sur (inicios de año), los alisios del noreste soplan con mucho más intensidad en toda la costa occidental. Esto se atribuye al desplazamiento hacia el Caribe de masas de aire polar, lo que provoca la aparición de centros de alta presión.

A pesar de que existen en el litoral caribeño de Honduras un número considerable de comunidades pesqueras, no existen trabajos sistemáticos que aborden, al menos parcialmente, los fenómenos físicos que suceden en dicha área, y que se puedan correlacionar posteriormente con el comportamiento observado de especies marinas ya incorporadas a la dieta cotidiana de la población local, o bien, de importancia comercial ligada a la creación de divisas.

Con los resultados del presente trabajo se pretende hacer un aporte al conocimiento de las principales características hidrográficas de este cuerpo de agua, con la finalidad de entender mejor los posibles cambios sufridos por este ecosistema en los últimos años y sus impactos en la actividad pesquera y acuícola.

\section{MATERIALES Y MÉTODOS}

Con los auspicios del Proyecto PREPAC (Plan Regional de Pesca y Acuicultura Continental) se realizó un levantamiento hidrográfico en 27 estaciones (Figura 1). Los muestreos mensuales se extendieron de octubre del 2005 a mayo del 2006. Su duración fue de un día y se llevaron a cabo en los primeros quince días de cada mes y en pleamar. Con una MINISONDE SURVEYOR4 de HYDROLAB de registro interno, se determinaron la temperatura $\left({ }^{\circ} \mathrm{C}\right)$ $\left( \pm 0.01^{\circ} \mathrm{C}\right)$, el pH $( \pm 0.2$ unidades $)$, el oxígeno disuelto $(\mathrm{mg} / \mathrm{L}) \quad( \pm 0.2$ $\mathrm{mg} / \mathrm{L})$, la salinidad ( \pm 0.2 unidades) y la turbidez (NUT) ( $\pm 1-5 \%)$. Considerando lo somero de la laguna $(2.1 \mathrm{~m}$ 
promedio y $7 \mathrm{~m}$ máximo, en el canal principal), los parámetros se tomaron en la superficie y a cada metro hasta el fondo. Los datos fueron procesados en SURFER 8 para realizar el graficado correspondiente.

\section{RESULTADOS Y DISCUSIÓN}

\section{Temperatura}

Debido a lo somero de la Laguna Los Micos, se hizo solamente el análisis del estrato superficial. La figura 2 presenta las distribuciones espaciales de la temperatura en el estrato superficial, de octubre del 2005 a mayo del 2006.

La temperatura a dicho nivel durante los ocho meses de muestreo osciló entre los 24.2 y $31.8^{\circ} \mathrm{C}$, siendo que las variaciones temporales extremas durante dicho período fueron del orden de los $7^{\circ} \mathrm{C}$. De modo general, los valores más bajos de este parámetro se ubican al final y principio de año (octubre-febrero), coincidiendo con el invierno del hemisferio norte, cuando los vientos alisios del noreste son intensos y persistentes en todo el Caribe. Simultáneamente, la Zona de Convergencia Intertropical (ZCIT) se localiza en su posición más al sur, y los centros de alta presión sobre el Golfo de México y el Mar Caribe dominan las condiciones meteorológicas de la región. Esta intensificación en el campo de vientos refuerza los procesos de mezcla en toda la columna de agua, actuando directamente sobre el campo térmico superficial. La transferencia de energía cinética de dichos vientos al cuerpo de agua provoca condiciones de isotermia en prácticamente toda la columna de agua. La figura 3A muestra un perfil vertical de temperatura correspondiente a febrero del 2006 en la estación 13, ubicada en el centro del sistema lagunar. Las diferencias térmicas entre la superficie y el fondo son de $1^{\circ} \mathrm{C}$. Resultados similares fueron reportados por Brenes y Ballestero (2008) para la Laguna de Perlas en el Caribe de Nicaragua.

Adicionalmente, las características del campo térmico superficial observadas en el área estudiada están en concordancia con el comportamiento de las aguas costeras adyacentes (Brenes et al. 1998).

A partir de marzo se observa un aumento progresivo de la temperatura superficial con un máximo centrado en abril. Nuevamente, el análisis vertical del campo térmico en la estación 13 (Figura 3B) para dicho mes muestra que el gradiente vertical térmico entre la superficie y el fondo no excedió nunca $0.3^{\circ} \mathrm{C} / \mathrm{m}$.

Como suele suceder en la mayoría de lagunas costeras, las regiones internas y someras presentan los mayores valores de temperatura, y la zona más externa y profunda, donde la conexión con el mar abierto es más fuerte, muestra las temperaturas más bajas. Noviembre fue un caso excepcional, debido a los efectos de la Tormenta Tropical Gamma, la cual 
Figura 2
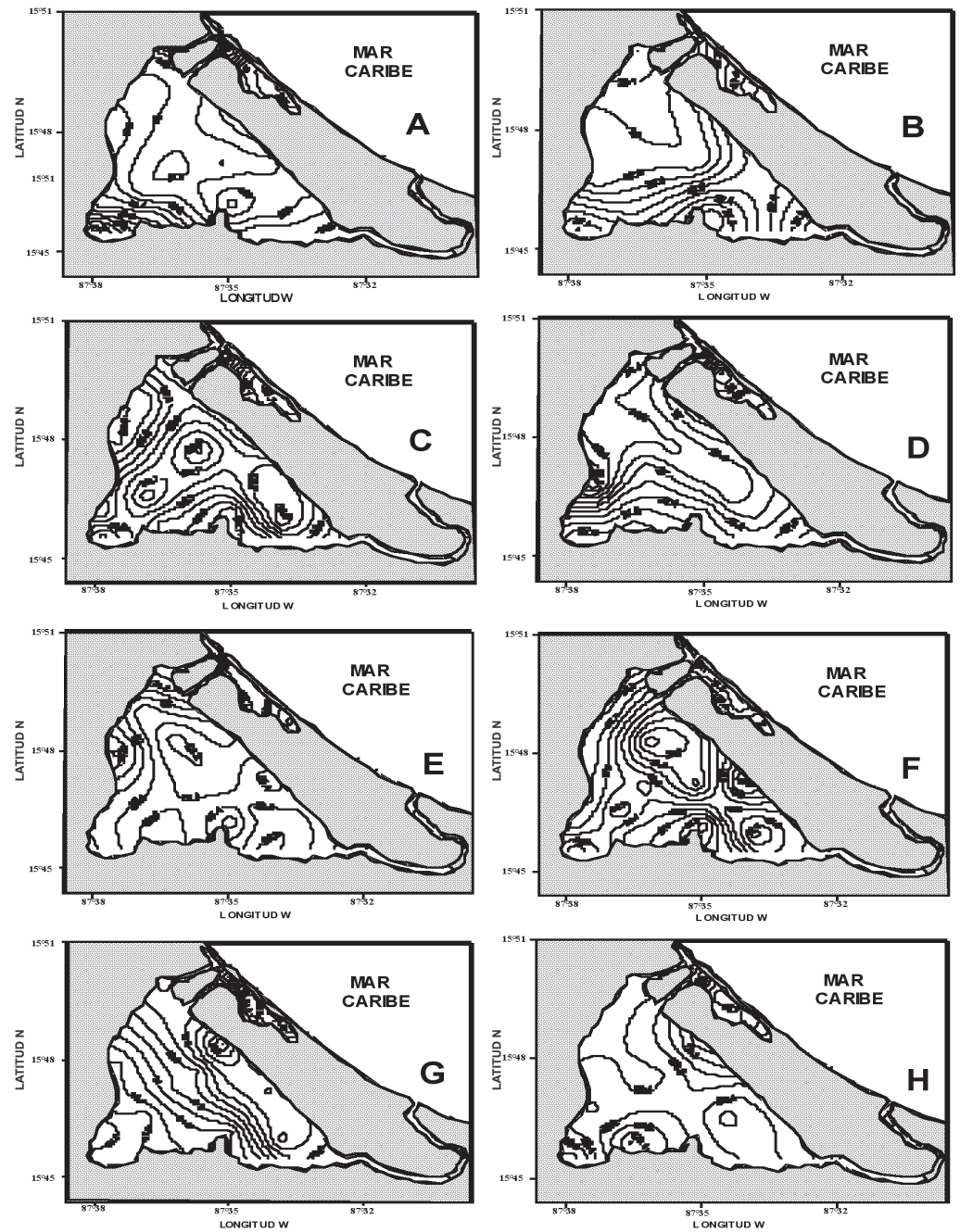

Distribuciones espaciales de la temperatura superficial $\left({ }^{\circ} \mathrm{C}\right)$ en la Laguna Los Micos-Quemada. Octubre (A), noviembre (B), diciembre (C), enero (D), febrero (E), marzo (F), abril (G) y mayo $(\mathrm{H})$.

Spatial distributions of surface temperature $\left({ }^{\circ} \mathrm{C}\right)$ in Los Micos-Quemada Lagoon. October (A), November (B), December (C), January (D), February (E), March (F), April (G) and May (H). 
Figura 3

FEBRERO 2006
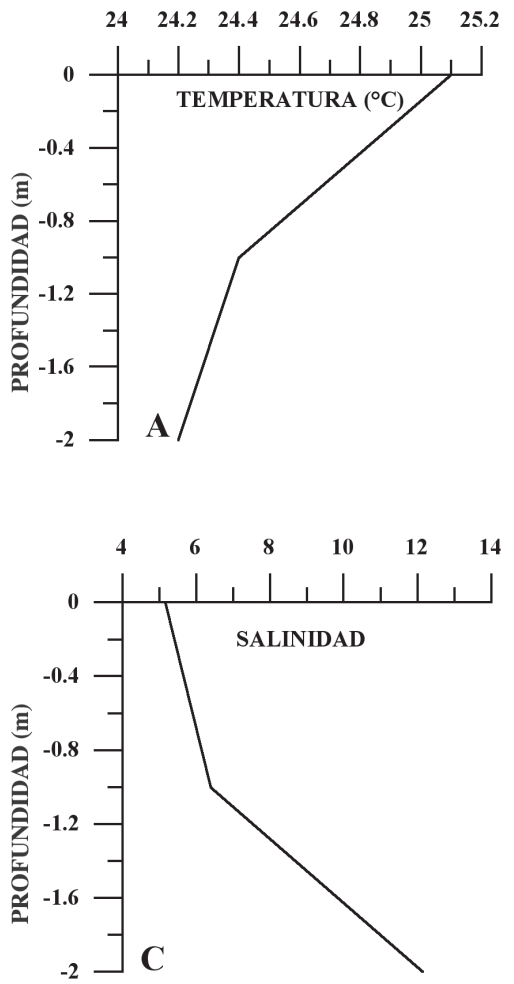
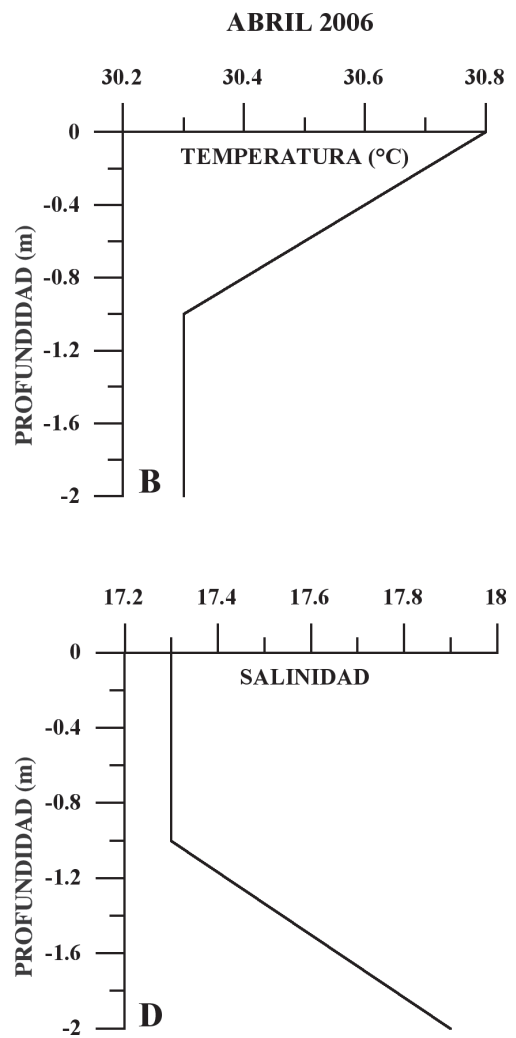

Perfiles verticales de temperatura $\left({ }^{\circ} \mathrm{C}\right)$ y salinidad para los meses de febrero (A y C) y abril (B y D) en la estación 13.

Vertical profiles of temperature $\left({ }^{\circ} \mathrm{C}\right)$ and salinity. February (A and C) and April (B and D) at station number 13.

aumentó considerablemente el aporte de agua dulce por escorrentía, sobre todo a través de los ríos San Alejo y La Esperanza en la ribera sureste de la laguna. En este mes cerca de la Barra de Miami la temperatura fue ligeramente mayor.

\section{Salinidad}

En la figura 4 se observa la distribución espacial de la salinidad super- ficial, en la cual los valores oscilaron entre 2 y 27. Los valores más altos se ubican en los lugares más cercanos a la costa, donde la interacción con las aguas costeras adyacentes es más fuerte, disminuyendo hacia el interior de la laguna.

La salinidad superficial presenta un marcado ciclo estacional, con valores superiores a 12 durante los meses secos en prácticamente todo el cuerpo de agua, mientras que en 
Figura 4
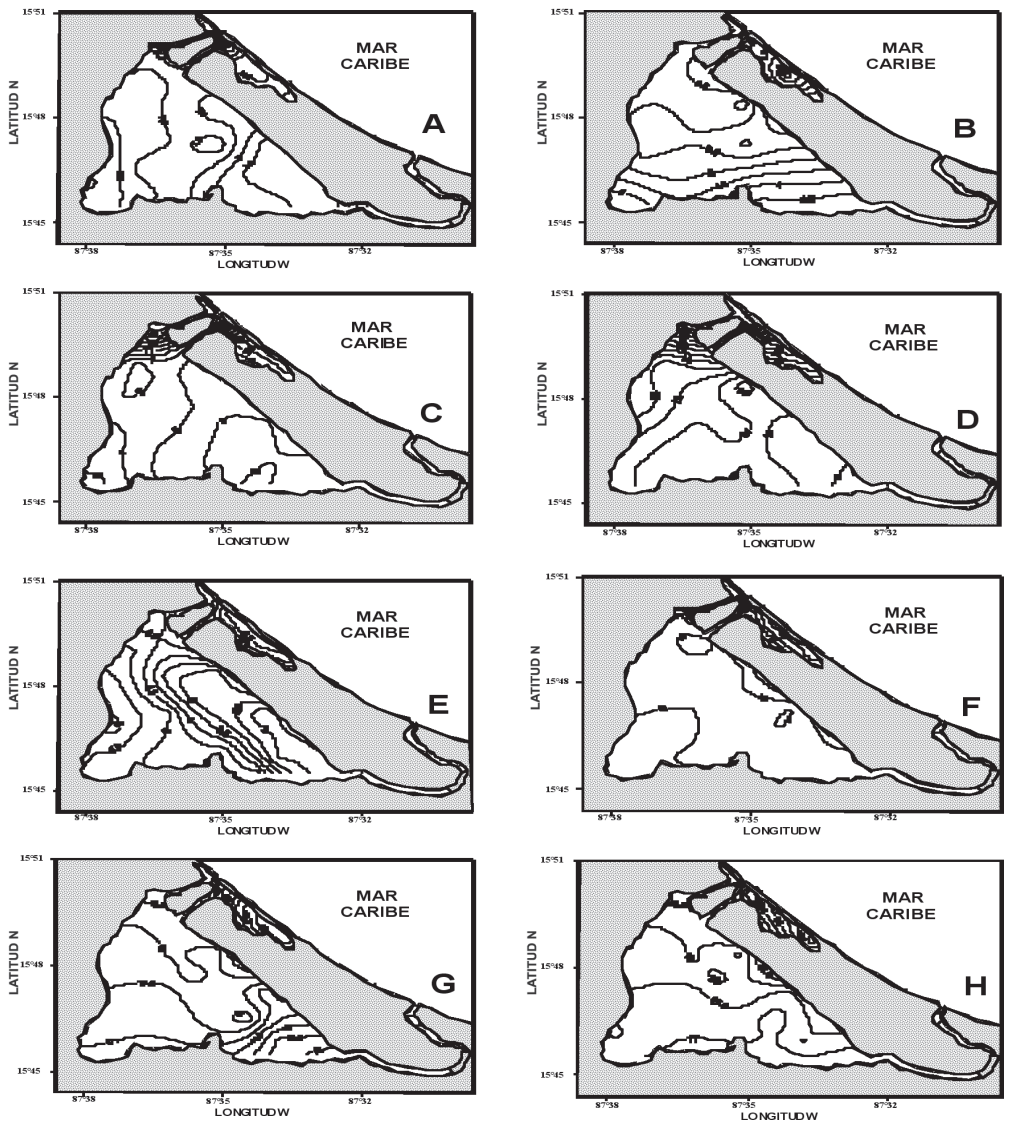

Distribuciones espaciales de la salinidad superficial en la Laguna Los Micos-Quemada. Octubre (A), noviembre (B), diciembre (C), enero (D), febrero (E), marzo (F), abril (G) y mayo (H). Spatial distribution of surface salinity (ups) in Los Micos-Quemada Lagoon. October (A), November (B), December (C), January (D), February (E), March (F), April (G) and May (H).

la época lluviosa (octubre a febrero) están alrededor de 6 (excepto en enero del 2006). Esto obedece a que los procesos de dilución están fuertemente influenciados por la descarga de varios ríos que vierten sus aguas en dicha laguna, principalmente por los ríos San Alejo y La Esperanza.
Estos ríos, a su vez, presentan una variación temporal en sus caudales asociada al ciclo anual de precipitaciones en esta zona. En la figura 3C se observa un fuerte gradiente vertical para el mes de febrero en la estación 13. Las diferencias salinas entre el estrato superior y el fondo alcanzan 
el valor de 8 , con un gradiente vertical cercano a $4.5 \mathrm{~m}^{-1}$. En los meses de diciembre y enero se observaron fuertes gradientes espaciales de salinidad en el área inmediata a la Barra de Miami, lo cual hace pensar en una influencia importante de la onda mareal sobre los procesos de mezcla en ese período.

El máximo salino en superficie ocurre entre abril y mayo, en fase con el máximo de temperatura. Este máximo salino se extiende a lo largo de toda la laguna. La parte más externa mantiene un rango de salinidades entre 22 y 18 , mostrando un carácter más oceánico.

Con el cierre de la barra en marzo, la disminución de las precipitaciones y el aumento de la evaporación, las diferencias de salinidad entre la superficie y el fondo se reducen a 0.70 , como puede observarse en la figura $3 \mathrm{D}$. La poca profundidad de la Laguna Quemada hace que presente salinidades mayores que la Laguna Los Micos, debido probablemente a un efecto de evaporación y porque carece de tributarios directos que le aporten agua dulce.

Durante el período de lluvias (octubre-febrero), la precipitación mensual se sitúa entre 250 y $440 \mathrm{~mm}$ para un total anual de 2.500-2.800 mm (Zúñiga, 1990), no obstante, entre el 14 y el 22 de noviembre del 2005, el paso de la Tormenta Tropical Gamma dejó un acumulado de casi $1.100 \mathrm{~mm}$ sobre la zona de estudio. Este exceso de precipitación se ve reflejado en las distribuciones espaciales de la turbi- dez, como se discutirá más adelante En estos meses se observa un fuerte gradiente espacial entre las áreas cercanas a la barra, y las salinidades en casi todo el interior de la laguna son inferiores a 8. La forma de las isohalinas evidencia un predominio del agua dulce proveniente principalmente del Río La Esperanza (su desembocadura se localiza en la ribera suroeste de la laguna cerca de la estación 24), sobre el agua salada que está siendo transportada hacia la laguna.

\section{pH}

Los valores de $\mathrm{pH}$ encontrados para el período de estudio se ubicaron entre los 7.8 y 8.7 (Figura 5), y son propios de una laguna costera sometida a la influencia directa de las aguas marinas. Los valores más altos se localizan en la zona central del sistema lagunar y los más bajos en las zonas cercanas a las orillas, debido a la degradación de materia orgánica y al aporte de los tributarios. Durante todo el período de muestreo no se presentaron variaciones importantes del $\mathrm{pH}$, sin embargo, los gradientes espaciales más evidentes ocurrieron durante los meses de mayor precipitación: octubre, diciembre y febrero.

\section{Turbidez}

La turbidez como una propiedad óptica de partículas en suspensión en el agua es notoria en aguas costeras $y$, particularmente, en estuarios y la- 
Figura 5
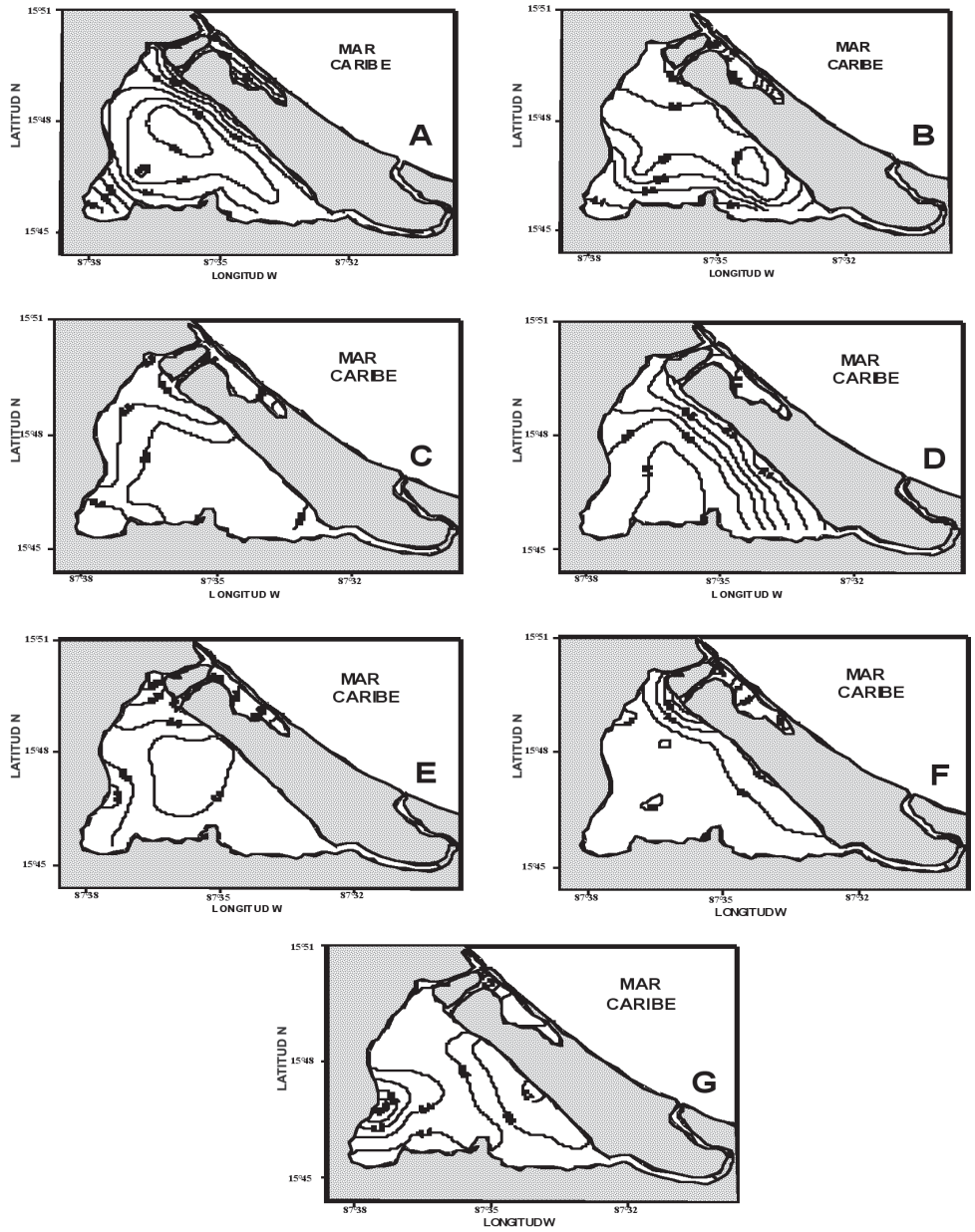

Distribuciones espaciales del pH superficial en la Laguna Los Micos-Quemada. Octubre (A), diciembre (B), enero (C), febrero (D), marzo (E), abril (F) y mayo (G).

Spatial distribution of surface $\mathrm{pH}$ in Los Micos-Quemada Lagoon. October (A), December (B), January (C), February (D), March (E), April (F) and May (G).

gunas costeras donde varía ampliamente a lo largo del año, alcanzando un máximo durante las inundaciones de la estación lluviosa, sin embargo, casi siempre está presente. Existe información acerca de su influencia sobre los organismos acuáticos, de- bido a su efecto sobre el descenso de oxígeno, además se ha visto que la mayoría de las especies de peces prefieren aguas claras para desarrollarse (Contreras et al. 1997).

En cuanto a la turbidez (Figura 6), los máximos valores se obtuvieron 
Figura 6
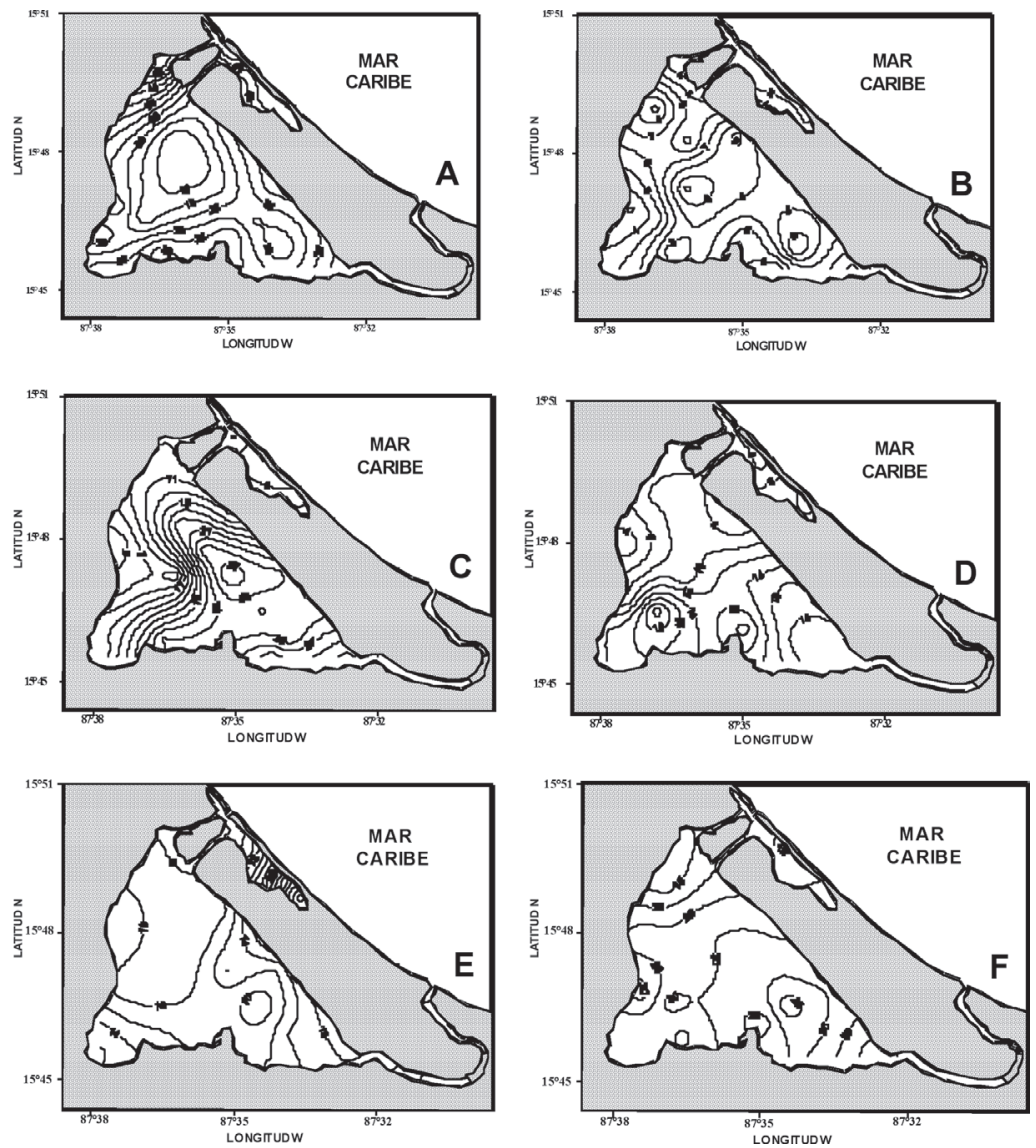

Distribuciones espaciales de la turbidez superficial (NUT's) en la Laguna Los Micos-Quemada. Diciembre (A), enero (B), febrero (C), marzo (D), abril (E) y mayo (F).

Spatial distribution of surface turbidity (NUT's) in Los Micos-Quemada Lagoon. December (A), January (B), February (C), March (D), April (E) and May (F).

en diciembre y febrero. La zona con aguas más claras a través del período de estudio se localiza de manera general en la región cercana a la barra, excepto en diciembre (Figura 6A), mes en el cual también la Laguna Quemada muestra valores superiores a 40 NUT, debido a la influencia de las aguas costeras inmediatas, las cuales probablemente retenían aún los efectos de las altas descargas de los ríos a causa de las precipitaciones que afectaron la zona por la Tormenta Tropical Gamma dos semanas atrás. La transparencia del agua es importante en las áreas con vegetación acuática y animales, y el papel más importante en cuanto al grado de 
transparencia en un ecosistema acuático surge del efecto de material en suspensión que impide o permite la mayor o menor entrada de luz, y que presenta una atención especial en relación con la productividad primaria y el flujo de energía en la comunidad (Reid \& Word, 1976).

La turbidez en la Laguna Los Micos no muestra cambios importantes durante todo el período de muestreo, oscilando entre 30 y 5 NUT. Como se comentó previamente, la excepción se presentó en diciembre del 2005, cuando la turbidez en la laguna alcanzó niveles muy elevados $(>35$ NUT), debido aún a los posibles efectos de la Tormenta Tropical. En enero y marzo del 2006, la turbidez presentó sus menores valores, a partir de mayo comenzó a aumentar nuevamente con el inicio del período lluvioso.

\section{Oxígeno disuelto}

La variación temporal del oxígeno disuelto muestra que los máximos y mínimos para todo el período de estudio se localizaron en octubre y mayo, respectivamente (Figura 7).

La turbulencia, las corrientes, las actividades biológicas, la salinidad y la temperatura juegan un papel importante en la distribución del contenido de oxígeno dentro de la laguna. Los mayores valores de oxígeno superficial obtenidos entre octubre y febrero están asociados a procesos de turbulencia generados por la intensi- ficación del campo de vientos en la región de estudio y a las bajas temperaturas del agua observadas en esa misma época. Los mayores gradientes espaciales se observan en octubre (Figura 7A). A partir de marzo, con el aumento de la temperatura de las aguas, se inicia una disminución significativa en los niveles de este parámetro.

Al cerrarse la barra en el mes de marzo se produjo una caída del oxígeno que persiste hasta el mes de mayo, con una distribución más homogénea en el cuerpo de agua. Bajas concentraciones de oxígeno están relacionadas con una escasa circulación asociada a la falta de interacción con el mar adyacente, a aumentos de temperatura o a procesos de descomposición de materia orgánica, como por ejemplo en zonas cercanas a las orillas. Por otro lado, el descenso de la concentración de oxígeno también está relacionado con disturbios en el fondo lagunar, a consecuencia de la resuspensión de sedimentos.

\section{CONCLUSIONES}

La temperatura superficial durante los meses de muestreo osciló entre los 24.2 y $31.8^{\circ} \mathrm{C}$, con variaciones temporales extremas del orden de los $7^{\circ} \mathrm{C}$. Los valores más bajos de este parámetro se ubican al final y principio de año (octubre-febrero).

La salinidad superficial presenta un ciclo estacional bien definido, con valores superiores a 12 durante los 
Figura 7
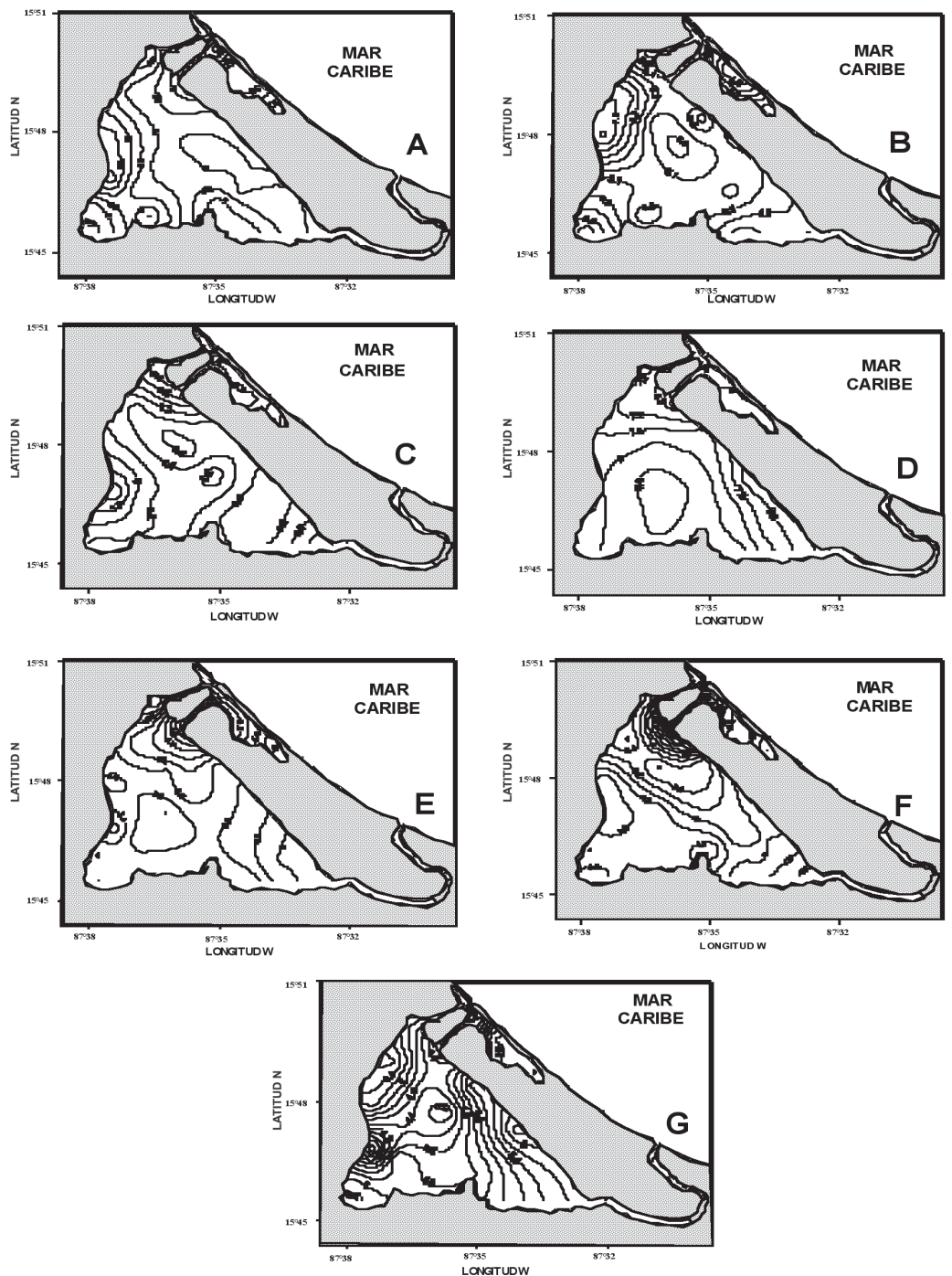

Distribuciones espaciales del oxígeno disuelto superficial (mg/L) en la Laguna Los Micos-Quemada. Octubre (A), diciembre (B), enero (C), febrero (D), marzo (E), abril (F) y mayo (G).

Spatial distribution of surface oxygen $(\mathrm{mg} / \mathrm{L})$ in Los Micos-Quemada Lagoon. October (A), December (B), January (C), February (D), March (E), April (F) and May (G). 
meses secos en prácticamente todo el cuerpo de agua, mientras que en la época lluviosa (octubre a febrero) están alrededor de 6 .

Los valores de $\mathrm{pH}$ encontrados para el período de estudio se ubicaron entre los 7.8 y 8.7 y son propios de una laguna costera sometida a la influencia directa de las aguas marinas. Los valores más altos se localizan en la zona central del sistema lagunar y los más bajos en las zonas cercanas a las orillas, debido a la degradación de materia orgánica y al aporte de los tributarios.

En cuanto a la turbidez, los máximos valores se observan en febrero y diciembre. La zona con aguas más claras a través del período de estudio se localiza de manera general en la región cercana a la barra.

Los mayores valores del oxígeno superficial se localizaron entre octubre y febrero y los mínimos en mayo, en concordancia con la mayor turbulencia que caracteriza a la laguna durante el período de intensificación de los alisios del noreste en el primer caso, y con una disminución en los procesos de intercambio de energía entre la laguna y la zona costera adyacente en el segundo caso, como consecuencia del cierre de la Barra de Miami.

\section{AGRADECIMIENTOS}

Agradecemos a Mario González Recinos, Director del Proyecto PREPAC (Plan Regional de Pesca y Acui- cultura Continental), por facilitarnos la información hidrográfica obtenida en la Laguna Los Micos-Quemada en el Caribe de Honduras. Este estudio fue financiado parcialmente por la Universidad Nacional de Costa Rica (Laboratorio de Oceanografía y Manejo Costero).

\section{BIBLIOGRAFÍA}

Brenes, C. L \& Ballestero, D. (2008). Variability of thermohaline properties in Pearl Lagoon, Nicaragua. Revista UNICIENCIA, 22 $(1,2), 123-129$.

Brenes, C. L., Gallegos, A. y Coen, E. (1998). Variación anual de la temperatura superficial en el Golfo de Honduras. Rev. Biol. Trop., 46, Supl (4), 187-197.

Contreras, E. F., Castañeda, L. O., Torres, A. R. y Gutiérrez, M. F. (1997). Problemática sobre las lagunas costeras mexicanas II. Hidrología y productividad primaria. Contactos 3a Época, 20, 5-12. Gallegos, A. (1996). Descriptive Physical Oceanography of the Caribbean Sea. In: Marine Science and Sustainable Development, Edited by George Maul AGU. Coast. Estuar. Stud., 51, 36-55.

Gray, K. (1993). Regional Meteorology and Hurricanes. In: Edited by George A. Maul. Climatic Change in the Intra-Americas Sea (pp. 87-99). United Nations of Environmental Program.

Instituto Hondureño de Turismo. (1993). Estudio del impacto am- 
biental de la construcción de la carretera de acceso al proyecto turístico Bahía de Tela. Tegucigalpa, Honduras.

PREPAC. (2006). Caracterización del Sistema Lagunar Los Micos-Quemada con énfasis en la pesca y acuicultura. San Salvador, El Salvador.

PROLANSATE, Fundación para la Protección de Lancetilla, Punta
Sal e Texiguat. (2004). Plan de Manejo Parque Nacional Jeannette Kawas. Tela, Atlántida. Honduras.

Reid, G. K. \& Wood, R. D. (1976). Ecology of Inland Waters and Estuaries. D. Van Nostrand Company, New York, USA.

Zúniga, E. (1990). Las modalidades de la lluvia en Honduras. Editorial Guaymuras, Tegucigalpa, Honduras. 